\title{
Giant Vesical Calculus Formation as a Complication of Augmentation Cystoplasty
}

\author{
Manoj Kumar $^{1} \cdot$ Ranjeet Kumar Singh $^{1} \cdot$ Rakesh Kapoor $^{1}$
}

Received: 14 May 2015 / Accepted: 12 October 2015 /Published online: 12 November 2015

(C) Association of Surgeons of India 2015

\begin{abstract}
A 44-year-old female presented with the history of recurrent UTI and intermittent hematuria. She underwent augmentation ileocystoplasty for small capacity bladder 19 years back. Patient was on clean intermittent catheterization (CIC) since then. Abdominal radiograph and ultrasonography showed the large vesical calculus. Open cystolithotomy was done, and a yellowish brown hard stone weighing $1025 \mathrm{~g}$ was removed. Chemical analysis revealed struvite stone. Postoperative period was uneventful. Regular bladder wash, lifelong surveillance and follow-up is advisable.
\end{abstract}

Keywords Vesical calculus · Augmentation cystoplasty · Cystolithotomy $\cdot$ Giant bladder stone

A 44-year-old female presented with the history of recurrent UTI and intermittent hematuria. She underwent augmentation ileocystoplasty for small capacity bladder 19 years back. Patient was on clean intermittent

Manoj Kumar

drmanoj1611@yahoo.com

Ranjeet Kumar Singh

drranjeerkrsingh@gmail.com

Rakesh Kapoor

rkapoor@sgpgi.ac.in

1 Department of Urology and Renal Transplant, Sanjay Gandhi Postgraduate Institute of Medical Sciences, C-Block Urology Office, SGPGIMS Hospital, SGPGIMS, Lucknow 226014, India catheterization (CIC) since then. Abdominal radiograph and ultrasonography showed the large vesical calculus (Fig. 1). Open cystolithotomy was done, and a yellowish brown hard stone weighing $1025 \mathrm{~g}$ was removed (Fig. 2). Chemical analysis revealed struvite stone. Postoperative period was uneventful.

Successful augmentation has been reported by many investigators using bowel segments from the ileum, colon, and stomach. Complications such as recurrent UTIs, spontaneous perforation, bladder stones, and the potential for malignancy are commonly reported.

Stone formation in augmentation cystoplasty is one of the late but common complications. The literature reported incidence of bladder calculi after Augmentation cystoplasty varies between 10 and $52 \%[1,2]$. These calculi are usually

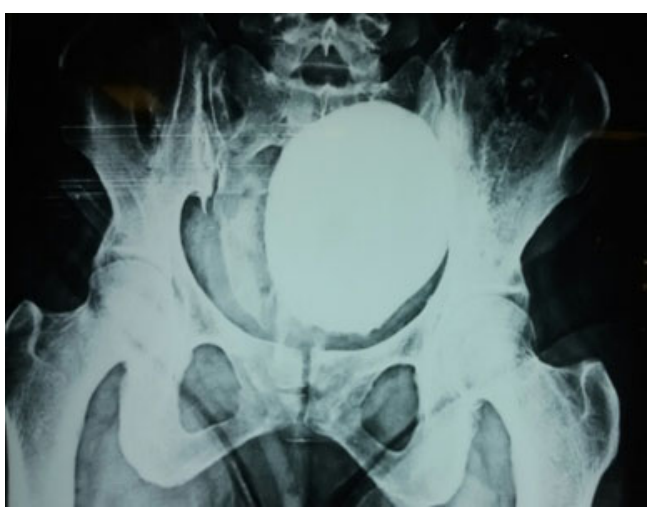

Fig. 1 Plain radiograph of kidney, ureter, and bladder showing large vesical calculus 


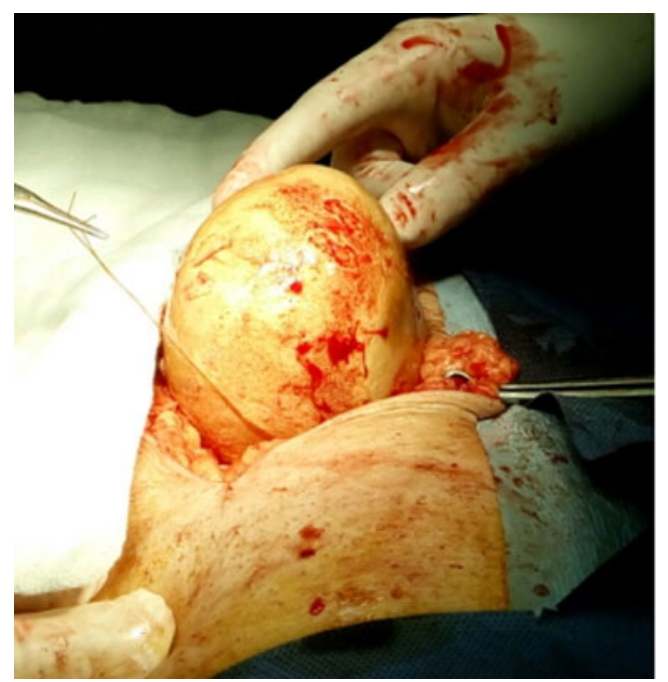

Fig. 2 Intraoperative photograph showing bladder calculus being removed from augmented bladder

composed of triple phosphate, calcium carbonate, and calcium oxalate. The various proposed etiological theories are copious mucous production acting as a nidus and urinary stasis with recurrent urinary tract infections, most commonly urea-splitting organisms in an alkaline environment [3-5]. Regular bladder wash, lifelong surveillance, and follow-up is advisable.

\section{Compliance with Ethical Standards}

Ethical Approval All procedures performed in studies involving human participants were in accordance with the ethical standards of the institutional and/or national research committee and with the $1964 \mathrm{Hel}-$ sinki Declaration and its later amendments or comparable ethical standards.

Conflict of Interest The authors declare that they have no competing interests.

\section{References}

1. Austin JC (2008) Long-term risks of bladder augmentation in pediatric patients. Curr Opin Urol 18(4):408-412

2. Veeratterapillay R, Thorpe AC, Harding C (2013) Augmentation cystoplasty: contemporary indications, techniques and complications. Indian J Urol 29(4):322-327

3. Mathoera RB, Kok DJ, Nijman RJ (2000) Bladder calculi in augmentation cystoplasty in children. Urology 56:482

4. Rink RC (1999) Bladder augmentation. Options, outcomes, future. Urol Clin N Am 26:111

5. Kumar S, Jayant K (2015) Massive vesical calculi formation as a complication of augmentation cystoplasty. Nephrourol Mon 7(1): e22297 